\title{
ERCC1 and topoisomerase I expression in small cell lung cancer: Prognostic and predictive implications
}

\author{
MARIA SERENO $^{1 *}$, PALOMA CEJAS $^{2 *}$, VÍCTOR MORENO ${ }^{2}$, CRISTÓBAL BELDA-INIESTA $^{2}$, \\ ROCIO LÓPEZ ${ }^{2}$, MANUEL NISTAL ${ }^{3}$, JAIME FELIU ${ }^{2}$ and JAVIER DE CASTRO CARPEÑO ${ }^{2}$ \\ ${ }^{1}$ Department of Medical Oncology, Infanta Sofía Hospital, Paseo Europa 34, 28702 San Sebastian De Los Reyes; \\ Departments of ${ }^{2}$ Medical Oncology and ${ }^{3}$ Pathology, La Paz University Hospital, \\ Paseo de la Castellana 261, 28046 Madrid, Spain
}

Received November 21, 2011; Accepted January 17, 2012

DOI: 10.3892/ijo.2012.1378

\begin{abstract}
Small cell lung cancer is the most aggressive lung cancer subtype. The standard treatment approach is based on cisplatin regimens. Although response rates to treatment are approximately $60-80 \%$, the median survival is still very poor. Excision repair cross complementation group 1 (ERCC1) is an enzyme that removes cisplatin-induced DNA adducts and has been related with prognosis and cisplatin response. Topotecan is the standard treatment as second-line therapy and it is an inhibitor of topoisomerase I (TOP I). We selected 76 patients with small cell lung (SCLC) to analyze the ERCC1 and TOP I mRNA expression. ERCC1 was studied both by quantitative PCR and immunohistochemistry. A significant association was found between the inmunohistochemistry expression of ERCC1 and the lack of platinum response $(\mathrm{p}=0.001)$. Moreover, low levels of TOP I RNA were shown to be linked to cisplatin response $(\mathrm{p}=0.002)$. In the survival analysis, a significant correlation between a better PFS with a low TOP I RNA expression as well as a negative ERCC1 inmunostaining were found, in both cases with a significant $\mathrm{p}$-value ( $\mathrm{p}=0.02$ and 0.009 , respectively). In summary, our results suggest the use of ERCC1 immunohistochemistry and TOP I mRNA analysis to predict cisplatin response and prognosis in SCLC patients.
\end{abstract}

Correspondence to: Dr Javier De Castro Carpeño, Department of Medical Oncology, La Paz University Hospital, Paseo de la Castellana 261, 28046 Madrid, Spain

E-mail: jcastro.hulp@salud.madrid.org

Dr Maria Sereno Moyano, Department of Medical Oncology, Infanta Sofía Hospital, Av Europa 37, 28702 San Sebastian de los Reyes, Madrid, Spain

E-mail: mariasereno75@gmail.com

${ }^{*}$ Contributed equally

Key words: small cell lung cancer, excision repair cross complementation group 1, topoisomerase I, cisplatin response, prognosis

\section{Introduction}

Small cell lung cancer (SCLC) is the most aggressive lung cancer subtype, with a strong tendency for early dissemination as well as high frequency of metastasis. SCLC accounts for $14 \%$ of new lung cancer cases diagnosed in USA and Europe (1) and staging determines prognosis and treatment. The standard treatment approach to patients with limited stage (SCLC) and good performance status is a combination of chest radiation and chemotherapy based on cisplatin (P) and etoposide (E), which results in complete response rate of $50-80 \%$ and a 5 -year survival probability of $12-25 \%$ (2). In case of extensive disease (ED), the main treatment is a combination of cisplatin with either etoposide or irinotecan. Reponses rates are approximately $60-80 \%$ and the median survival reaches 7-12 months.

Cisplatin causes monoadducts and intrastrand or interstrand cross-links in DNA $(3,4)$. Nucleotide excision repair plays a central role among DNA repair pathways and has been associated with resistance to cisplatin-based chemotherapy. The excision repair cross complementation group 1 (ERCC1) enzyme, plays a rate-limiting role in the nucleotide excision repair pathway which recognizes and removes cisplatininduced DNA adducts (5). The role of ERCC1 in resolving DNA interstrand cross-link-induced double-strand breaks has been clearly shown (6-8). Various studies have reported the relationship between ERCC1 expression and the effect of cisplatin-induced DNA adducts in human ovarian cancer cells in vitro (9), in primary gastric adenocarcinomas (10), colorectal cancer (11) and, more recently, in esophageal cancer (12). Pivotal data from primary non-small cell lung cancer (NSCLC) specimens have suggested a link between ERCC1 immunoexpression and cisplatin resistance. However, for NSCLC untreated patients, a high ERCC1 expression in selected cases was associated with a better survival $(13,14)$. Taken together, all these studies show that ERCC1 expression level could be inversely associated with cisplatin response. However in terms of prognosis, high levels of ERCC1 expression are a favourable prognostic factor of survival in some tumors like NSCLC. Nevertheless, the value of ERCC1 as cisplatin predictor and as prognosis marker in SCLC has scarcely been explored with only two previous studies showing contradictory results $(15,16)$. 
Topotecan and irinotecan are topoisomerase I (TOP I) inhibitors that are active in the treatment of chemotherapynaive and chemotherapy-sensitive patients with recurrent SCLC. Topoisomerases are enzymes capable of altering DNA topography for the purposes of relieving torsional strain during processes like replication, transcription, and recombination. These enzymes facilitate passing one strand of DNA through a break in the opposing strand (type I subfamily) or passing a duplex from the same or different molecule through a double stranded gap (type II subfamily). Topoisomerases can relax either negative supercoils, or both positive and negative supercoils of the DNA. These unique features are needed because of the double helical structure of DNA, in which TOPOs help access stored information for transcription, recombination and replication purposes (17).

Topotecan $(\mathrm{T})$ is the standard treatment as second-line setting for patients with chemotherapy-sensitive disease (durable response to first line therapy of at least 3 months), with response rate of $19-37 \%$. In addition, topotecan has been studied as doublet and triplet combination regimens to help assess its potential in first-line treatment of SCLC. Topotecan has shown preliminary results of $75 \%$ of objective responses in combination with paclitaxel (18), 90\% with paclitaxel and carboplatin, (19) $81 \%$ with etoposide and carboplatin, (20) and 67\% with ifosfamide (21). Although TOP I inhibitors have attracted extensive interest in the treatment of SCLC, there are no studies analyzing the role of changes in TOP I expression in this tumor.

Resistance to chemotherapy is the main cause of poor outcome in patients with SCLC (22). Therefore, the identification of markers that may identify those patients who would benefit from the current chemotherapy or chemoradiotherapy (CRT) approaches has strong clinical implications. To this aim, we retrospectively analyzed the ERCC1 and TOPO I expression as predictors of response and survival in a SCLC patients cohort treated with platinum-based combination chemotherapy.

\section{Patients and methods}

Patients and samples. Between January 2000 and December 2007, 228 patients were diagnosed with SCLC. Of these clinicopathological data and adequate tissue were available in 76 (33.3\%) patients; therefore, this group was selected for the study. All patients were treatment naive. Tissue samples were taken from their primary tumor by bronchoscopic or thoracoscopic biopsy in 73 cases and from the metastatic sites (bone marrow) in three patients. The Veterans Administration Lung Study Group system was used for staging patients (22).

The most commonly administered chemotherapy regimen was EP in 53 cases (etoposide $100 \mathrm{mg} / \mathrm{m}^{2}$, cisplatin $25 \mathrm{mg} / \mathrm{m}^{2}$ on days 1-3; 50 patients), followed by carboplatin-etoposide in 22 patients (carboplatin AUC 5 y 6 etoposide on day 1 and etoposide $100 \mathrm{mg} / \mathrm{m}^{2}$, on days 1-3). Chemotherapy was repeated every 3 weeks. Among all the patients who underwent chemotherapy, 19 received 4 cycles and 54 patients received 6 cycles of an etoposide-platinum combination. In patients with ED SCLC, two to four additional cycles of chemotherapy were administered if a response was achieved after two cycles of chemotherapy. Thoracic concurrent irradiation (45 Gy) was performed in patients with limited disease and it was started with the second-third courses of chemotherapy on the same day.
Among second line chemotherapy regimens, the most common treatment used was CAV (cisplatin, adriamycin and vincristine) in $31.5 \%$ followed by paclitaxel $(21 \%)$ and topotecan $(10 \%)$. Fifteen patients received chemotherapy and the most common regimen administered was topotecan $(11.8 \%$ of all the patients included) (Table I).

Response to treatment was evaluated with chest CT after the third and sixth course of chemotherapy in ED SCLC, and after the concurrent chemo-radiation treatment in limited stage (LD), according to the World Health Organization criteria (23). Patients were defined platinum-resistant when time to progression after EP was less than 3 months. After the completion of treatment, patients were evaluated with chest CT every 3 months for the first and second year, every 6 months for the next 3 years, and yearly thereafter.

ERCC1 immunohistochemistry. Bronchoscopic lung biopsies were fixed in $4 \%$ buffered formaldehyde and embedded in paraffin (FFPE). Four-micrometer-thick sections were cut and stained with hematoxylin and eosin for pathological review. The study was approved by the institutional review board of the hospital. Gene expression analysis was performed blinded to the clinico-pathological data.

Sections of $4 \mu \mathrm{m}$ were cut from formalin-fixed, paraffinembedded tissue blocks from the afore-mentioned patients. Slides were de-paraffined and endogenous peroxidase activity was blocked by incubation in $3 \% \mathrm{H}_{2} \mathrm{O}_{2}$ in methanol for $10 \mathrm{~min}$ at room temperature (RT). Antigens were retrieved by incubation in EDTA for $45 \mathrm{~min}$ at $155^{\circ} \mathrm{C}$. The primary mouse anti-human monoclonal antibodies against ERCC1 (ERCC1 Ab-2, Neomarkers, Fremont, CA, USA) was diluted at 1:100 in $1 \% \mathrm{BSA}$ in TBS. Tissue slides were incubated with the antibody for $30 \mathrm{~min}$ at RT. Slides were then rinsed in TBS and incubated with the peroxidase-based EnVision ${ }^{\mathrm{TM}}$ kit (Dako Corp., Carpinteria, CA, USA) for $30 \mathrm{~min}$ at RT. Afterwards, specimens were incubated with diaminobenzidine chromogenic substrate (Dako Corp.) for $5 \mathrm{~min}$ at RT. Sections were counterstained with hematoxylin, stepwise dehydrated through graded alcohols and cleared in xylene.

Immunohistochemical score. The negative controls for these proteins were made by omission of the primary antibody during the process of immunohistochemical staining. The positive controls for ERCC1 were the presence of staining in the non-tumor bronchial mucosa. The slides were examined independently by two expert surgical pathologists blinded to both clinical and pathologic data. Discordant results were discussed and agreed on by both pathologists. Immunohistochemical staining was quantified using a visual grading system based on the extent of staining (percentage of positive tumor cells) according to the grading system of Soria et al. It was considered positive when more than $10 \%$ of the cells showed nuclear staining (24).

ERCC1 and TOP I quantitative PCR. RNA isolation was performed from the formalin-fixed paraffin-embedded bronchoscopic lung biopsies. Ten $(5 \mu \mathrm{m})$ sections from each endoscopic specimen block were placed into a microcentrifuge tubes. One additional 4- $\mu \mathrm{m}$ section was stained with hematoxylin and eosin (H\&E). Paraffin was removed by xylene extraction, and RNA was isolated using a commercially available kit: 
Table I. Base characteristics of the patients.

\begin{tabular}{lc}
\hline Characteristics of the patients & Number $(\%)$ \\
\hline Age (years) & 62 \\
Range & $43-81$ \\
& \\
Gender & \\
Male & $66(86)$ \\
Female & $10(14)$ \\
& \\
Stage & \\
Limited & $27(35)$ \\
Disseminated & $49(65)$ \\
& \\
First line chemotherapy & $76(100)$ \\
Platinum/etoposide & $76(100)$ \\
Second line chemotherapy & \\
Cisplatin/adriamycin/vincristine & $49(64.7)$ \\
Paclitaxel & $25(31.5)$ \\
Topotecan & $16(21)$ \\
Third line chemotherapy & $8(10)$ \\
Paclitaxel & $15(19.7)$ \\
Topotecan & $5(6.5)$ \\
Gemcitabine-vinorelbine & $9(11.8)$ \\
Cheatment modality in limited stage & $1(1.3)$ \\
Concomitant chemo-radiation & \\
\hline
\end{tabular}

Epicentre Technologies Inc. (Madison, WI), MasterPure RNA Purification kit according to the manufacturer's instructions. Total RNA was measured spectrophotometrically, measurements were performed in UltraPure destillated water DNAse, RNAse free (Gibco, Invitrogen). Reverse transcription of RNA derived from FFPE material was performed from $250 \mathrm{ng}$ of total RNA of each case and $125 \mathrm{U}$ Multiscribe reverse transcriptase (High-Capacity cDNA Archive Kit, Applied Biosystems, Foster City, CA, USA). Random primers were used to primer cDNA synthesis. Relative cDNA quantitation for both ERCC1 and TOPO 1 against the reference gene ( $\beta$-actin) was done using a fluorescence-based real-time detection method [ABI PRISM 7900 Sequence Detection System (TaqMan); Applied Biosystems]. We profiled expression of the three genes ERCC1 (Hs Hs01012158_m1 assay), TOP I (Hs01052825_m1) and the internal reference ACTH (Hs99999903m1 assay) (in triplicates). These TaqMan primers and probes are designed to produce amplicons of around 100 nucleotides. ERCC1 and TOP I Gene expression levels are expressed as ratios (differences between the $C_{t}$ values) between two absolute measurements (genes of interest/internal reference gene).

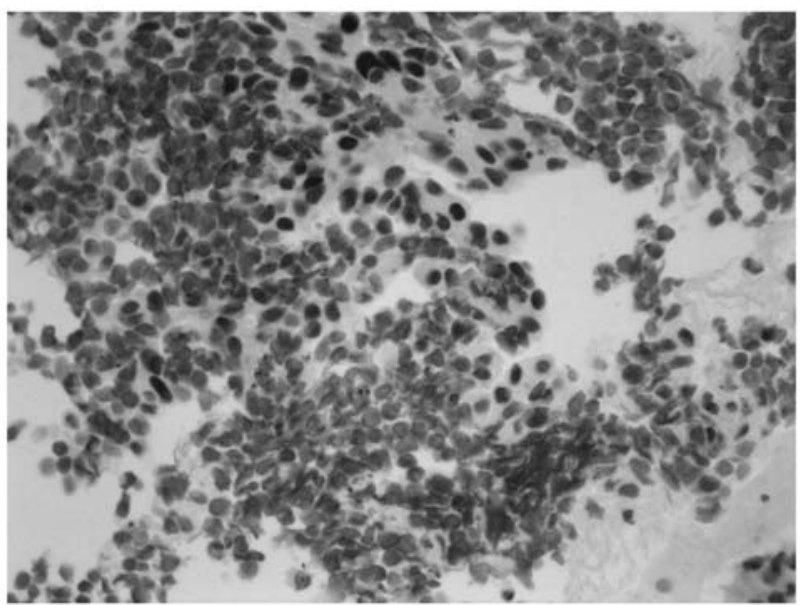

Figure 1. Positive inmunohistochemical expression of ERCC1 in a small cell lung cancer sample.

Statistical analysis. Overall survival (OS) and progression-free survival (PFS) was calculated using the Kaplan-Meier method. The differences between the survival curves were tested by using the log-rank test.

The Cox proportional-hazards regression model was used to determine the joint effects of several variables on survival. A comparison of clinico-pathological characteristics was evaluated with Fisher's exact test. Cox's proportional hazards multivariate analysis was used to evaluate which of the significant factors at the univariate analysis had a significant influence on survival. Statistical significance was set at $\mathrm{p}=0.05$. All analyses were performed with SPSS for Windows 13.0 software.

\section{Results}

Patient characteristics. The clinico-pathological characteristics of the 76 patients are listed in Table I. Of the patients $49(65 \%)$ had an ED whereas 27 (35\%) had LD. All received chemotherapy based on PE with concurrent radiation in LD. Among the 76 patients selected, $43(51.3 \%)$ were considered platinum responders and $33(48.7 \%)$ platinum resistant, according to the definition previously given.

ERCC1 expression and cisplatin response. Positive immunoexpression of ERCC1 was observed in 29 (52\%) whereas in 24 patients the ERCC1 immunostaining was negative $(45.3 \%)$ (positive immunostaining of ERCC1; Fig. 1). Twenty-six cases could not be evaluated for the immunohistochemistry and PCR analysis. A significant association was found between negative immunohistochemistry expression of ERCC1 and the platinum response ( 7 patients in the cisplatin-resistant group showed negative staining vs 31 patients in the cisplatin responders group, 12.5 vs $87.5 \%$ respectively, $\mathrm{p}=0.001$ ) (Fig. 2).

The analysis of ERCC1 mRNA by qPCR showed low ERCC1 expression in 24 cases $(50 \%)$ and 17 cases showed a high ERCC1 expression. Among low expression cases, there were 27 patients considered cisplatin responders $(70.8 \%)$. In contrast, among the 30 non-cisplatin responder patients, 10 patients (41\%) had high ERCC1 expression. There was a correlation between low expression of ERCC1 and cisplatin response that did not reach statistical significance $(\mathrm{p}=0.54)$ (data not shown). 


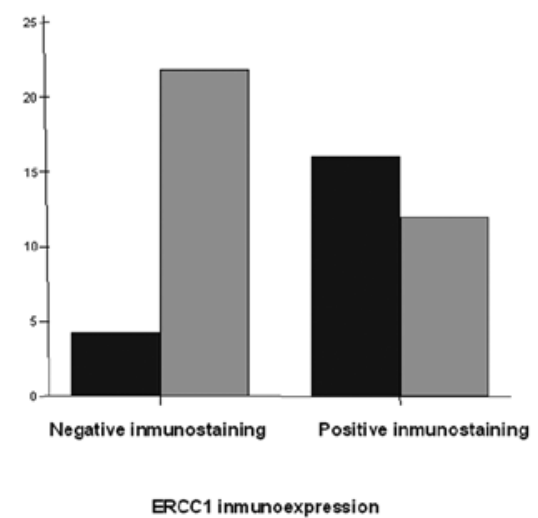

Figure 2. Correlation between ERCC1 inmunoexpression and cisplatin response.

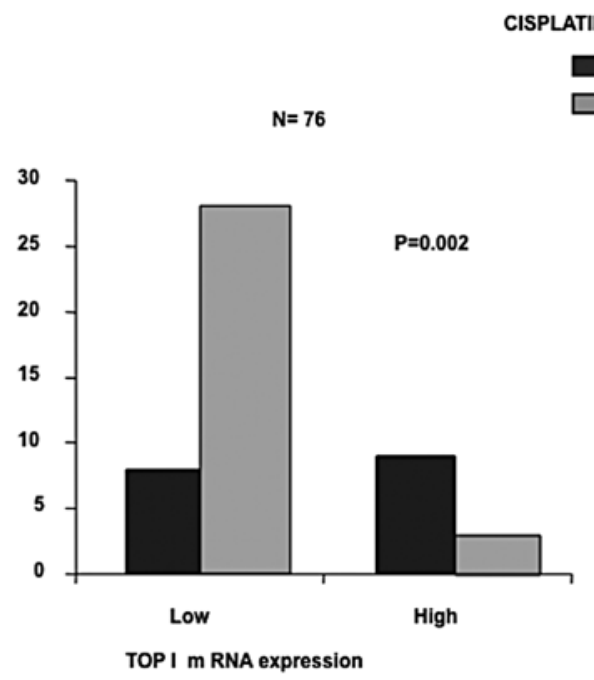

Figure 3. Correlation between cisplatin response and mRNA expression of ERCC1.

TOP I expression and cisplatin response. Only 48 cases could be evaluated for the PCR analysis because of problems of availability of the samples. We observed an opposite relationship between TOP I RNA expression levels and the response to cisplatin: 28/36 (77.8\%) patients in the responder group showed a low level expression of TOP I expression, whereas 9 patients (75\%) patients in the resistant group showed high expression of TOP I (p=0.02) (Fig. 3).

ERCCI and TOP I expression and prognosis. After a median follow-up time of 12 months, the median PFS was 6 months (CI 95\%, 5-6.9 months) and OS was 9 months (CI 95\%, 7.4-10.6) (Fig. 4).

Patients with negative ERCC1 immunostaining showed a lower risk of PFS compared to cases with positive ERCC1 immunostaining HR=0.456 (CI 95\% 0.256-0.819; $\mathrm{p}=0.009$ ) (Fig. 5a). In contrast, the qPCR analysis of ERCC1 did not show the risk of PFS, with only a trend towards a lower risk of progression in patients with low ERCC1 mRNA levels HR=0.641 (CI 95\% $0.35-1.15 ; \mathrm{p}=0.14$ ) (Fig. 5b).

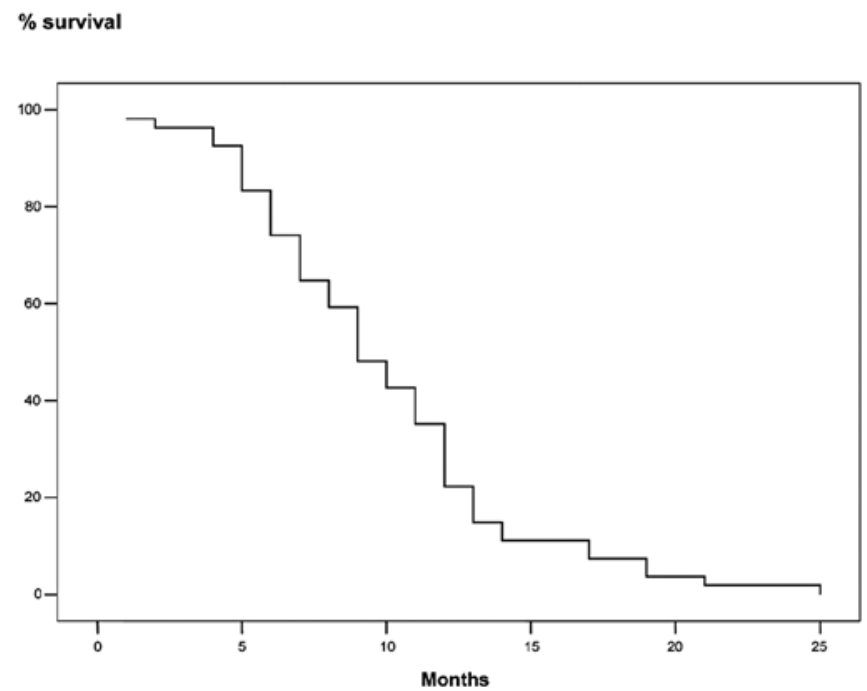

Figure 4. Kaplan Meyer overall survival curve.

Table II. Univariate analysis of progresion-free survival (PFS).

\begin{tabular}{lc}
\hline Variable & P-value \\
\hline Stage & 0.09 \\
$\quad$ Limited & \\
Extensive & \\
Gender & 0.96 \\
$\quad$ Male & \\
Female & \\
Response to cisplatin based-chemotherapy & $0.0001^{\mathrm{a}}$ \\
$\quad$ Positive & \\
Negative & \\
ERCC1 inmunoexpression & $0.009^{\mathrm{a}}$ \\
$\quad$ Low & \\
High & \\
ERCC1 mRNA expression & \\
Low & 0.14 \\
High & \\
TOP I mRNA expression & \\
Low & \\
High &
\end{tabular}

Patients with low TOP I mRNA expression showed a significantly lower risk of progression compared to those patients with high TOP I expression HR=0.446 (CI 95\% 0.223-0.888; p=0.02) (Fig. 5c).

We also analyzed for a possible correlation between TOP I and ERCC 1 expression using Pearson correlation, but we could not demostrate an association $(S q r$ lineal $=0.036)$. The results of the univariate analysis are shown in Table II. A multivariate analysis was performed including gender, stage, cisplatin response, ERCC1 and TOP I expression. Response to cisplatin 
$\mathbf{a}$
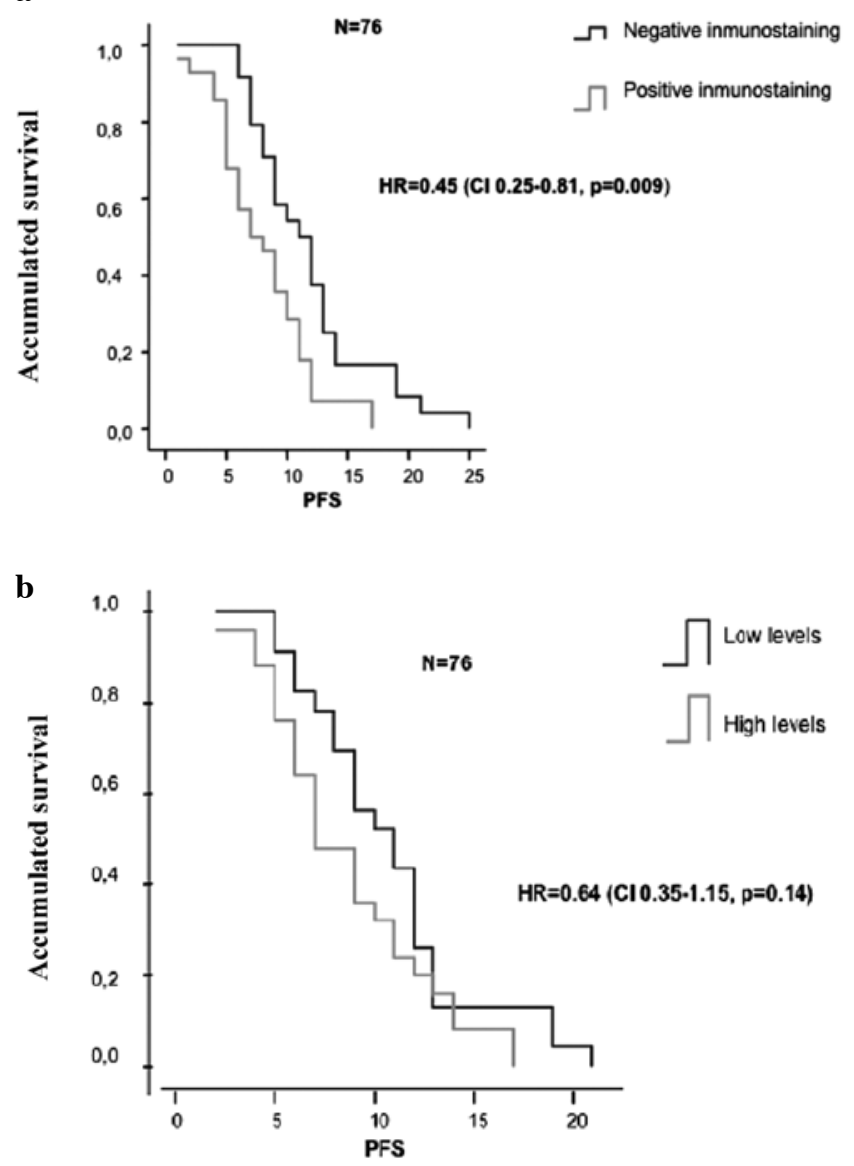

c

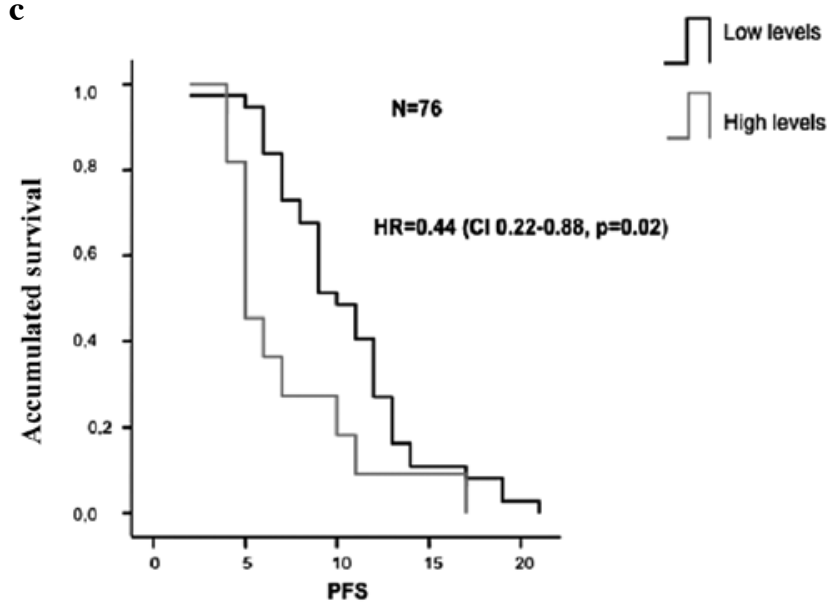

Figure 5. (a) Correlation between ERCC1 inmunostaining and PFS. (b) Association of PFS and mRNA ERCC1 levels. (c) Correlation of mRNA TOP I levels and PFS.

as well as TOP I expression were the parameters with the most relevant impact on survival. Cisplatin response reached statistical significance in the multivariate analysis $(\mathrm{p}=0.003)$ but TOP I expression showed only a trend with a statistical significance in the first case I expression ( $\mathrm{p}=0.06$ ) (data not shown).

\section{Discussion}

In this study, we explored the relationship between ERCC1 and TOP I expression and cisplatin-response and PFS in patients diagnosed with SCLC. ERCC1 status has been related to cisplatin response in other tumors such as NSCLC (22-24), bladder cancer (25) and head and neck cancer $(26,27)$. However, scarce data have been reported with regard to SCLC, which represents one of the most aggressive cancer types. Moreover, SCLC therapy has not experienced significant improvements despite active research in this field in recent years. Several reports have demonstrated that alterations in DNA damage repair proteins are associated with resistance to cisplatin based chemotherapy or radiotherapy (25-27). Cisplatin resistance is one of the most important causes of treatment failure in SCLC (28). In this study we analyzed ERCC1 expression in relation to cisplatin response. This potential relationship could help us to select those patients with a potential cisplatin response and those with primary resistance. In these resistant cases, cisplatin administration could be avoided; decreasing associated platinum toxicity in patients with a positive ERCC1 expression. Our group showed a highly significant relationship between ERCC1 expression and cisplatin resistance as analyzed by IHC ( $\mathrm{p}=0.001)$. These findings have a direct influence on survival: patients with ERCC1 positive staining had a significant worse PSF compared to those patients with negative staining $(\mathrm{p}=0.009)$. Moreover, when ERCC1 was analyzed by QPCR, it did not reach statistical significance. One possible explanation for this discordance could be the insufficient sample size or the lack of linear correlation between mRNA and protein expression. In this sense, our study is the first to compare ERCC1 expression both by QPCR and immunohistochemistry and the results point to immunohistochemistry as the method of choice for analysis of ERCC1.

There are two published studies that have analyzed ERCC1 expression and PFS in SCLC $(15,16)$. Lee et al analyzed ERCC1 immunoexpression in tumor specimens from 77 patients with SCLC who were treated with a platinum regimen. The authors found that in LD patients, high expression of ERCC1 was an independent prognostic factor for poor OS $(p=0.046)$, along with male gender $(\mathrm{p}=0.033)$ (15). Along the same lines, Ceppi et al analyzed ERCC1 RNA expression in 85 patients, also showing that ERCC1 was an independent prognostic factor for survival in LD patients; however these results could not be reproduced in ED (16). Consistent with the two previous studies, we have shown that tumor stage and ERCC1 immunoexpression were the only variables with significant impact on PFS, $\mathrm{p}=0.04$ and 0.006, respectively. In contrast to the results of Ceppi et al, neither gender nor stage could be analyzed in our study due to the unbalanced sample size. This could also be the reason why the stage did not reach the statistical significance in univariate analysis.

Apart from ERCC1, in this report TOP I expression was also explored. An interesting relationship between low TOP I RNA expression and a better cisplatin response and PFS was found. The multivariate analysis showed that TOP I expression had a significant influence on PFS, as well as cisplatin response, but in the first case the association did not reach statistical significance. There is no previous evidence regarding the role of TOP I in survival nor in cisplatin prediction in SCLC. However, there is controversy regarding the prognosis value of TOP I in other types of tumors (29-33). Tsavaris et al found that levels of expression of TOP I were higher in malignant cells from tumor recurrences compared to primary tumors, suggesting a role of TOP I in tumor recurrence (34), but these 
results could not be confirmed by Paradiso et al (35). TOP $\mathrm{I}$ is the target of topotecan and irinotecan, which have been explored mainly as second line treatments, and recently, in a first line setting (32). Our study is the first to demonstrate a positive relationship between low levels of TOP I and better cisplatin response as well as a longer PFS in SCLC patients. TOP I plays a crucial function in DNA replication (36-39). Low levels of TOP I indicate inappropriate DNA replication, therefore, a lower rate of proliferation. Perhaps a high level of TOP I is a surrogate marker of proliferation and could be a prognostic factor as well as a predictive marker to response to chemotherapy, in this case, to cisplatin. A recent study by Kohara et al demonstrated in preclinical data, responses to TOP I inhibitors in platinum-resistant cells (40). In this study, we were not able to analyze the relationship between TOP I expression and topotecan or irinotecan treatment because only $10 \%$ of the patients received topotecan as second line treatment, and none received topotecan as first line treatment. The reason is that at the beginning of recruitment, topotecan was not yet approved for SCLC treatment in Spain. Further studies will be developed to explore a potential relationship between TOP I expression and response with contradictory results (37). Maden et al found that higher levels of TOP I correlated with sensitivity to TOP I inhibitors (38); however on the other hand, MacLeod et al reported that gene copy number and protein expression are inversely correlated with sensitivity to SN 38, an inroinotecan metabolite, in vitro in several breast and colon tumor cell lines (39).

Based on our results, we suggest that there is a positive impact of the low levels of TOP I mRNA on PFS, but these findings should be confirmed in further studies. We identified a profile of patients with SCLC with negative ERCC1 immunostaining as well as low mRNA levels of TOP I with a good cisplatin response as well as a longer PSF. Mukai et al reported similar results in different tumors using immunohistochemistry and PCR analysis in tissue specimens of breast, gastric and nonsmall cell lung cancer. They found that high levels of ERCC1 and TOP I mRNA are related to recurrence (37).

In addition to this prognostic information, our results could be the first step in designing other studies with a large patient sample sizes to validate these findings. They may suggest a therapeutic algorithm for first line treatment based on ERCC1 and TOP I mRNA expression/levels. Those patients with poor ERCC1 expression and low levels of mRNA of ERCC1 could be treated with a platinum regimen. However, if ERCC1 immunohistochemistry is positive or mRNA levels are high, cisplatin resistance is indicated, and in these cases we could analyze TOP I levels. According to previously reported data, high levels of TOP I may be involved in TOP I inhibitors (topotecan and irinotecan) response, but low levels would be associated with a poor response. In this study, it was not possible to report a correlation with TOP I inhibitor treatment, therefore, we are working on further studies to explore this aspect.

\section{Acknowledgements}

We thank Dr Miguel Angel García-Cabezas and Dr Manuel González Barón for expert pathological and clinical assistance.

\section{References}

1. Jackman DM and Johnson BE: Small cell lung cancer. Lancet 366: 1385-1396, 2005.

2. Sandler AB: Chemotherapy for small cell lung cancer. Semin Oncol 30: 9-25, 2003.

3. Lawley PD and Phillips DH: DNA adducts from chemotherapeutic agents. Mutat Res 355: 13-40, 2004.

4. Phyllip P and Mallen H: Chemotherapy in non-small cell lung cancer: a meta-analysis using updated data on individual patients from 52 randomised clinical trials. Br Med J 311: 899-909, 2001.

5. Mu D, Hsu DS and Sancar A: Reaction mechanism of human DNA repair excision nuclease. J Biol Chem 271: 8285-8294, 1996.

6. Niedernhofer LJ, Odijk H, Budzowska M, van Drunen E, et al: The structure-specific endonuclease Ercc1-Xpf is required to resolve DNA interstrand crosslink-induced double-strand breaks. Mol Cell Biol 24: 5776-5778, 2004.

7. Sancar A: Mechanisms of DNA excision repair. Science 266: 1954-1956, 1994.

8. Zamble DB, Mu D, Reardon JT, et al: Repair of cisplatin-DNA adducts by the mammalian excision nuclease. Biochemistry 35 : 10004-10013, 1997.

9. Li Q, Yu JJ, Mu C, Yunmbam MK, et al: Association between the level of ERCC-1 expression and the repair of cisplatin-induced DNA damage in human ovarian cancer cells. Anticancer Res 20: 645-652, 2002.

10. Metzger R, Leichman CG, Danenberg KD, et al: ERCC1 mRNA levels complement thymidylate synthase mRNA levels in predicting response and survival for gastric cancer patients receiving combination cisplatin and fluorouracil chemotherapy. $\mathrm{J}$ Clin Oncol 16: 309-316, 1998.

11. Shirota Y, Stoehlmacher J, Brabender J, et al: ERCC1 and thymidylate synthase mRNA levels predict survival for colorectal cancer patients receiving combination oxaliplatin and fluorouracil chemotherapy. J Clin Oncol 19: 4298-4304, 2001.

12. Joshi MB, Shirota Y, Danenberg KD, et al: High gene expression of TS1, GSTP1, and ERCC1 are risk factors for survival in patients treated with trimodality therapy for esophageal cancer. Clin Cancer Res 11: 2215-2221, 2005.

13. Lord RV, Brabender J, Gandara D, et al: Low ERCC1 expression correlates with prolonged survival after cisplatin plus gemcitabine chemotherapy in non-small cell lung cancer. Clin Cancer Res 8: 2286-2291, 2002.

14. Simon GR, Sharma S, Cantor A, et al: ERCC1 expression is a predictor of survival in resected patients with non-small cell lung cancer. Chest 127: 978-983, 2007.

15. Lee H, Han H, Kim JH, et al: Expression of excision repair cross complementation group 1 protein predicts poor outcome in patients with small cell lung cancer. Lung Cancer 59: 95-104, 2004.

16. Ceppi P, Longo M, Volante $\mathrm{M}$, et al: Excision repair cross complementing- 1 and topoisomerase II gene expression in smallcell lung cancer patients treated with platinum and etoposide: a retrospective study. J Thorac Oncol 3: 583-589, 2008.

17. Chhatriwala $\mathrm{H}$, Jafri $\mathrm{N}$ and Salgia R: A review of topoisomerase inhibition in lung cancer. Cancer Biol Ther 5: 1600-1607, 2006.

18. Jett JR, Hatfield A, Bauman M, et al: Phase II trial of topotecan and paclitaxel (TP) with G-CSF support alternating with etoposide and cisplatin (EC) in previously untreated extensive stage small cell lung cancer (ED-SCLC): a North Central Cancer Treatment Group study. Lung Cancer 29: 52-57, 2007.

19. Hainsworth JD, Gray JR, Burris HA, et al: Paclitaxel, carboplatin and topotecan as initial therapy for small cell lung cancer. Lung Cancer 29: 40, 2000.

20. Gillenwater HH, McCune JS, Donahue AE, et al: Phase I trial of topotecan (T), carboplatin, and etoposide (E) for extensive stage small cell lung cancer. Lung Cancer 29: p67, abs. 127, 2000.

21. Ventriglia M, Cabrera L, Maidana M, et al: A phase II trial of topotecan (Hycamtin) with ifosfamide (Duvaxan) plus mesna (Mesnex) as first line treatment inpatients (pts) with small cell lung cancer (SCLC. A preliminary report. Lung Cancer 29: p67, abs. 218, 2000.

22. Zelen M: Keynote address on biostatistics and data retrieval. Cancer Chemother Rep 3: 31-34, 1973.

23. Miller AB, Hoogstraten B, Staquet M, et al: Reporting results of cancer treatment. Cancer 47: 207-214, 1973.

24. Olaussen KA, Dunant A, Fouret P, et al: DNA repair by ERCC1 in non-small-cell lung cancer and cisplatin-based adjuvant chemotherapy. N Engl J Med 355: 983-991, 2006. 
25. Bellmunt J, Paz-Ares L and Cuello M: Gene expression of ERCC1 as a novel prognostic marker in advanced bladder cancer patients receiving cisplatin-based chemotherapy. Ann Oncol 18: 522-528, 2007.

26. Quintela-Fandino M, Hitt R, Medina PP, et al: DNA-repair gene polymorphisms predict favorable clinical outcome among patients with advanced squamous cell carcinoma of the head and neck treated with cisplatin-based induction chemotherapy. J Clin Oncol 24: 4333-4339, 2006.

27. Handra-Luca A, Hernandez J, Mountzios G, et al: Excision repair cross complementation group 1 immunohistochemical expression predicts objective response and cancer-specific survival in patients treated by Cisplatin-based induction chemotherapy for locally advanced head and neck squamous cell carcinoma. Clin Cancer Res 13: 38-55, 2007.

28. Reed E: ERCC1 and clinical resistance to platinum-based therapy. Clin Cancer Res 11: 6100-6102, 2005.

29. Recka M, Grotha M, Buchholzb E, et al: Topotecan and etoposide as first- line therapy for extensive disease small cell lung cancer: a phase II trial of a platinum-free regimen. Lung Cancer 48 : 409-413, 2005.

30. Hanna N, Bunn PA Jr, Langer C, et al: Randomized phase III trial comparing irinotecan/cisplatin with etoposide/cisplatin in patients with previously untreated extensive-stage disease smallcell lung cancer. J Clin Oncol 24: 2038-2043, 2006.

31. Noda K, Nishiwaki Y, Kawahara M, et al: Irinotecan plus cisplatin compared with etoposide plus cisplatin for extensive small-cell lung cancer. N Engl J Med 346: 85-91, 2004.

32. Sorensen M, Lassen U, Jensen PB, et al: Phase II study of a 3-day schedule with topotecan and cisplatin in patients with previously untreated small cell lung cancer and extensive disease. J Thorac Oncol 3: 902-906, 2008.
33. Ohashi N, Fujiwara Y, Yamaoka N, et al: No alteration in DNA topoisomerase I gene related to CPT-11 resistance in human lung cancer. Jpn J Cancer Res 87: 1280-1287, 1996.

34. Tsavaris N, Lazaris A, Kosmas C, et al: Topoisomerase I and IIalpha protein expression in primary colorectal cancer and recurrences following 5-fluorouracil-based adjuvant chemotherapy. Cancer Chemother Pharmacol 64: 391-398, 2009.

35. Paradiso A, Xu J, Mangia A, et al: A topoisomerase-I, thymidylate synthase primary tumour expression and clinical efficacy of 5-FU/CPT-11 chemotherapy in advanced colorectal cancer patients. Int J Cancer 20: 252-258, 2004.

36. Rothenberg ML: Topoisomerase I inhibitors: review and uptodate. Ann Oncol 8: 837-855, 1997.

37. Mukai M, Sato S, Ninomiya H, et al: Sensitivity to CPT-11 and platinum derivatives of stage I/II node-negative breast, lung, and gastric cancer with occult neoplastic cells in lymph node sinuses. Oncol Rep 18: 33-39, 1997.

38. Madden KR and Champoux JJ: Over expression of human topoisomerase I in baby hamster kidney cells: hypersensitivity of clonal isolates to campthoteicinas. Cancer Res 52: 525-532, 1992.

39. McLeod HL and Keith WN: Variation in topoisomerase I gene copy number as a mechanism for intrinsic drug sensitivity. Br J Cancer 74: 508-512, 1996.

40. Kohara H, Massahiro T and Kiura K: Synergistic effects of topoisomerase I inhibitor, 7-etyl-10-hydroxycampthotecines and irradiation in a cisplatin-resistant human small cell lung cancer cell line. Clin Cancer Res 8: 287-292, 2002. 